\title{
Dysplasia Surveillance in Inflammatory Bowel Disease: A Cohort Study
}

\author{
Sofia Saraiva ${ }^{a}$ Isadora Rosa ${ }^{a}$ Joana Moleiro ${ }^{a}$ João Pereira da Silva ${ }^{a}$ \\ Ricardo Fonseca ${ }^{b}$ António Dias Pereira ${ }^{a}$ \\ ${ }^{a}$ Department of Gastroenterology, Instituto Português de Oncologia de Lisboa Francisco Gentil E.P.E. (IPOLFG), \\ Lisbon, Portugal; ${ }^{b}$ Department of Pathology, Instituto Português de Oncologia de Lisboa Francisco Gentil E.P.E. \\ (IPOLFG), Lisbon, Portugal
}

\section{Keywords}

Inflammatory bowel disease (IBD) - Ulcerative colitis .

Crohn's disease · Dysplasia · Surveillance

\section{Abstract}

Introduction: Patients with colonic inflammatory bowel disease (IBD) are at an increased risk for colorectal cancer (CRC), whereby surveillance colonoscopy is recommended. Aim: To study the clinical and endoscopic variables associated with dysplasia in IBD patients. Methods: A cohort study was conducted on IBD patients who were part of a colonoscopy surveillance program between 2011 and 2016. Results: A total of 342 colonoscopies were performed on 162 patients (105 with ulcerative colitis [UC] and 57 with Crohn's disease). Random biopsies were performed at least once on $81.5 \%$ of patients; $33.3 \%$ of the patients underwent chromoendoscopy (CE) at least once. Endoscopically resectable lesions were detected in 55 patients (34\%), and visible lesions deemed unfit for endoscopic resection were found in 5 patients (3.1\%). Overall, 62 dysplastic visible lesions (58 with lowgrade dysplasia and 3 with high-grade dysplasia) and 1 adenocarcinoma were found in 34 patients. Dysplasia in random biopsies was present in 3 patients, the yield of random biopsies for dysplasia being 1.85\%/patient (3/162), 1.75\%/ colonoscopy $(6 / 342)$, and $0.25 \% /$ biopsy $(9 / 3,637)$. Dysplasia detected in random biopsies was significantly associated with a personal history of visible dysplasia $(p=0.006)$. Upon univariate analysis, dysplasia was significantly associated with the type of IBD, the performance of random biopsies, and CE ( $p=0.016 / 0.009 / 0.05$, respectively). On multivariate analysis, dysplasia was associated with duration of disease. Conclusion: Our data confirm that patients with long-standing IBD, in particular UC, should be enrolled in dysplasia surveillance programs, and that performing $\mathrm{CE}$ and random biopsies seems to help in the detection of colonic neoplastic lesions.

(C) 2020 Sociedade Portuguesa de Gastrenterologia Published by S. Karger AG, Basel

\section{Vigilância de displasia na doença inflamatória intestinal: estudo coorte}

\section{Palavras chave}

DII · Colite ulcerosa · Doença de Crohn · Displasia · Vigilância

\section{(C) 2020 Sociedade Portuguesa de Gastrenterologia} Published by S. Karger AG, Basel

This article is licensed under the Creative Commons AttributionNonCommercial-NoDerivatives 4.0 International License (CC BYNC-ND) (http://www.karger.com/Services/OpenAccessLicense). Usage and distribution for commercial purposes as well as any distribution of modified material requires written permission.
Sofia Saraiva

Department of Gastroenterology, Instituto Português de Oncologia de Lisboa Francisco Gentil E.P.E. (IPOLFG)

Rua Prof. Lima Basto, PT-1099-023 Lisbon (Portugal)

asofia.saraiva@gmail.com 


\section{Resumo}

Introdução: Nos doentes com doença inflamatória intestinal (DII) está recomendada vigilância por colonoscopia para detetar e tratar lesões neoplásicas iniciais, dado o risco aumentado de cancro colo-rectal (CCR). O objetivo do trabalho foi estudar variáveis clínicas e endoscópicas associadas a displasia. Métodos: Estudo coorte - doentes com DIl integrados num programa de vigilância de displasia entre 2011-2016. Resultados: Um total de 342 colonoscopias foi realizado em 162 doentes, 105 com colite ulcerosa (CU) e 57 com doença de Crohn (DC). Foram efetuadas biopsias aleatórias (BA) em 81,5\% dos doentes (média: $27.5 \pm 6.4$ biopsias/colonoscopia) e 33.3\% realizaram cromoendoscopia. 55 doentes apresentaram lesões endoscopicamente ressecáveis e 5 doentes lesões irressecáveis. No total, em 34 doentes, foram identificadas 6 lesões displásicas visíveis (58 com displasia de baixo grau e 3 com displasia de alto grau) e um adenocarcinoma. Foi detetada displasia em BA em 3 doentes sendo o rendimento das $B A$ de $1.85 \%$ por doente (3/162), 1.75\% por colonoscopia (6/342) e $0.25 \%$ por biopsia $(9 / 3,637)$. A displasia em BA associou-se à história pessoal de lesões displásicas ( $p=0.006$ ). A presença de displasia associouse, na análise univariada, com: tipo de DII ( $p=0.016)$, realização de BA ( $p=0.009)$ e cromoendoscopia $(p=0.05)$. $\mathrm{Na}$ análise multivariada, verificou-se associação com a duração da doença. Conclusão: Doentes com Dll de longa duração deverão ser incluídos num programa de vigilância de displasia. A realização de cromoendoscopia e BA é útil na deteção de lesões displásicas do cólon.

C 2020 Sociedade Portuguesa de Gastrenterologia Publicado por S. Karger AG, Basel

\section{Introduction}

Patients with long-standing inflammatory bowel disease (IBD) are at an increased risk for developing colorectal cancer (CRC). The magnitude of the risk remains a continuing subject of debate. Early studies found that the cumulative risk of CRC for patients with ulcerative colitis (UC) was $2 \%$ at 10 years, $8 \%$ at 20 years, and $18 \%$ at 30 years [1]. This high-risk estimate was later considered to be an overestimation due to selection bias; cohorts were selected from tertiary referral centers with a disproportionally high percentage of patients with severe disease [2].

More recently, Beaugerie et al. [3] found that the standardized incidence ratio of CRC is 2.2 for all IBD patients and 7.0 for patients with long-standing extensive colitis.
Table 1. Proposed risk factors for dysplasia in IBD patients

Clinical risk factors

Disease duration, extent, and severity

Personal history of dysplasia

Primary sclerosing cholangitis

A family history of CRC

Endoscopic risk factors

Active disease

Colonic stricture

Postinflammatory polyps

Tubular appearance of colon

IBD, inflammatory bowel disease; CRC, colorectal cancer.

Moreover, an Australian study reported a CRC cumulative incidence of $1 \%$ at 10 years, $3 \%$ at 20 years, and $7 \%$ at 30 years in IBD patients [4].

Besides a more careful selection of patients included in these recent studies, the declining risk of CRC over time may also reflect the increased implementation of surveillance strategies and the introduction of more effective treatments. In fact, a 2012 Danish population-based study determined that the CRC risk among patients with UC was comparable to that of the general population over a 30-year period [5].

In clinical practice, it is essential to know which risk factors modulate the CRC risk in IBD patients, in order to identify the subgroups of patients who need closer surveillance or more intensive treatment. Both clinical and endoscopic factors have been described [6] (Table 1). Histological inflammation also seems to be an independent risk factor for CRC [4]. Based on the presence and number of these risk factors, guidelines recommend tailored surveillance colonoscopy protocols to detect and treat neoplastic lesions in patients with long-standing IBD [4]. Standard definition white-light endoscopy (WLE) with random biopsies in apparently nondysplastic mucosa plus biopsy of visible lesions has classically been the most used method for surveillance colonoscopy $[6,7]$.

However, accumulating evidence has shown that dysplasia detection on IBD surveillance colonoscopy can be increased by dye-spraying chromoendoscopy (CE) with targeted biopsies. In fact, both the SCENIC Consensus and the European Crohn and Colitis Organization guidelines state that CE is more effective than WLE with random biopsies and is therefore the preferred method $[4,8]$.

There is a consensus that there is a need for random biopsies when using standard-definition WLE $[4,7,8]$. However, the performance of random biopsies when us-
98

GE Port J Gastroenterol 2021;28:97-105 DOI: $10.1159 / 000510728$
Saraiva/Rosa/Moleiro/Pereira da Silva/ Fonseca/Dias Pereira 
ing CE remains controversial $[8,9]$. Their low diagnostic yield, along with the increase in procedure time and costs, has been used as an argument to discourage the use of random biopsies with CE $[6,8,10]$. On the other hand, Moussata et al. [10] recently published results that emphasize the importance of performing random biopsies in patients with a personal history of neoplasia, primary sclerosing cholangitis (PSC), and/or a tubular-appearing colon. Along with other studies $[6,11,12]$, they showed that random biopsies increase the number of neoplasia diagnoses when specific risk factors are present. Mirroring this controversy, in SCENIC, the panellists could not reach a consensus regarding random biopsies with CE [8].

The aim of this study was to determine the clinical and endoscopic variables associated with dysplasia in IBD patients who were part of a colonoscopy surveillance program.

\section{Material and Methods}

\section{Study Population}

This was a cohort study conducted at a single tertiary care center. All IBD patients who were part of a colonoscopy surveillance program between 2011 and 2016 were considered eligible.

Patients aged $\geq 18$ years with a diagnosis of Crohn's disease (CD) involving $>50 \%$ of the colon, or with UC extending above the rectum with a duration $>8$ years (or any duration in patients with a concomitant diagnosis of PSC) were included.

Exclusion criteria included patients with previous colonic resections (other than ileocecal) or a previous CRC diagnosis.

\section{Colonoscopy Protocol}

All patients enrolled in the study underwent colonoscopy during the study period. Surveillance intervals were based on the number and type of risk factors (Table 1) and were according to the European guidelines [4]. Patients with high-risk features had colonoscopies performed with a 1-year interval; patients with an intermediate risk had a 3-year interval, and those with a low risk had a 5 -year interval. CE was initially planned for all patients. Random biopsies were performed according to the endoscopist's decision.

Endoscopic procedures were performed using standard-definition colonoscopes (Olympus ${ }^{\circledR} 145$ or 160 series) with patients under sedation (mild sedation with midazolam or deep sedation with propofol, administered by an anesthesiologist, at the prescribing physician's discretion). All CE were performed by a single trained endoscopist. The remaining exams were performed by either the same endoscopist or, in a few cases, by another IBD expert.

The colonoscope was advanced to the cecum while the colonic mucosa was meticulously washed with the water-jet pump. On withdrawal, each segment (cecum, ascending colon, transverse colon, descending-sigmoid, and rectum) was sequentially examined for lesions. CE was performed when the quality of preparation was rated good on the Boston Bowel Preparation Scale (i.e., BBPS >5) and there was no active disease (Mayo subscore $<2$ in UC patients, simple endoscopic score [SES-CD] $<4$ in CD patients, or Rutgeerts score $<3$ in postsurgical CD patients) [13-15]. CE was performed using $0.04 \%$ methylene blue via a spray catheter inserted through the biopsy channel. Circumferential application of the dye was followed by reinsertion and careful visualization of the epithelial surface every $30 \mathrm{~cm}$. No spasmolytic drugs were used.

\section{Biopsy Protocol}

Visible mucosal lesions were classified as either unfit for endoscopic resection or endoscopically resectable. In the former, targeted biopsy specimens were obtained. In the latter, biopsy forceps, snare polypectomy, or endoscopic mucosal resection was performed, and 4 samples were taken from the flat perilesional surrounding mucosa. In most cases (at the endoscopist's discretion), 4 random biopsies were also performed every $10 \mathrm{~cm}$, independently of whether CE was used or not.

\section{Histopathological Evaluation}

Biopsies were analyzed by expert gastrointestinal tract pathologists. Neoplasia was classified according to the modified Vienna classification [16] into low-grade dysplasia (LGD), high-grade dysplasia (HGD), or adenocarcinoma (ADC).

Colectomy was discussed as a therapeutic option from the first dysplasia diagnosis in all patients.

\section{Statistics}

A descriptive analysis was performed, expressing quantitative variables as means \pm SD or median and IQR, and qualitative variables as $n(\%)$.

Univariate and multivariate analyses were performed to identify the clinical and endoscopic factors associated with dysplasia. The $\chi^{2}$ test, Fisher's exact test, and Mann Whitney U test were used to assess associations between the presence of dysplasia and various demographic, clinical, and endoscopic risk factors, as qualitative or quantitative variables, respectively. The risk factors included were sex, age, type of IBD, duration of the disease, disease extent in UC, a family or personal history of neoplasia/dysplasia, concomitant PSC, random biopsies or CE being performed, the type of IBD treatment, and the presence of colonic strictures or postinflammatory polyps.

Variables found to be associated with dysplasia in the univariate analysis were included in a binary logistic regression model. Odds ratios (ORs) with 95\% confidence intervals (CIs) were used to express the strength of the association.

A survival analysis was also performed with the Kaplan-Meier method, using the time from IBD diagnosis to the first dysplasia or CRC cancer diagnosis (event), or to death or the last registered clinical observation. Log-rank tests and a multivariate Cox regression were run to determine if there were differences in time to the event when $\mathrm{CE}$ and/or random biopsies were performed. All analyses were performed using SPSS v25 (SPSS, Inc., Chicago, IL, USA). A significance level of $p=0.05$ for bilateral testing was accepted.

\section{Results}

\section{Study Population}

A total of 162 patients were included in the study, 105 with UC (70.5\% had pancolitis) and 57 with CD. Only 6 
Table 2. Demographics and disease characteristics

$\begin{array}{lc}\text { Male sex } & 83(52.1) \\ \text { Age at diagnosis, years } & 36.8(13.5) \\ \text { Duration of IBD at the start of the program, years } & 11.8(8.9) \\ \text { Ulcerative colitis } & 105(64.8) \\ \quad \text { Pancolitis } & 70.5 \% \\ \text { Crohn's disease } & 57(35.2) \\ \text { Concurrent primary sclerosing cholangitis } & 6(3.7) \\ \text { A family history of CRC } & 18(11.1) \\ \text { A personal history of colorectal dysplastic lesions } & 6(3.7) \\ \text { Previous ileocecal resections } & 25(15.4) \\ \text { Medication } & \\ \quad \text { 5-aminosalicylates } & 95.7 \% \\ \quad \text { Thiopurines } & 55.6 \% \\ \text { Anti-TNF therapy } & 24.1 \%\end{array}$

Values express $n(\%)$ or mean $\pm \mathrm{SD}$, unless otherwise indicated. CRC, colorectal cancer; IBD, inflammatory bowel disease; TNF, tumor necrosis factor.

patients had concurrent PSC, 18 had a family history of CRC, and 6 had a personal history of colorectal dysplastic lesions.

Regarding medication (data collected when drugs were administered), the majority of patients $(95.7 \%)$ were or had been on 5-ASA; half (55.6\%) of the patients received thiopurines, and $24.1 \%$ were or had been on antiTNF- $\alpha$. These and other patient demographics and clinical characteristics are presented in Table 2.

\section{Endoscopic Features}

In the 6-year study period, a total of 362 colonoscopies were performed. The mean number of colonoscopies was $2.1 \pm 1.2$ per patient, and it was significantly higher in patients in whom at least a dysplastic lesion was found than in those without dysplasia $(2.5 \pm 1.4$ and $2.0 \pm 1.1$, respectively; $p=0.05$ ).

A random biopsy was performed at least once in $81.5 \%$ of the patients, with a mean $27.5 \pm 6.4$ biopsy samples per colonoscopy (samples taken from flat perilesional mucosa segments were not included in this analysis). Due to poor bowel preparation (in $>50 \%$ of the cases) or active inflammation at the time of the procedure, only one-third $(33.3 \%)$ of the patients underwent at least $1 \mathrm{CE}$, and 95\% had a random biopsy performed in the same procedure.

Endoscopically resectable lesions were detected in 55 patients (34\%), and visible lesions deemed unfit for endoscopic resection were found in 5 patients (3.1\%). Stenosis was documented in 6 patients (3.7\%), and postinflammatory polyps were found in 33 patients $(20.4 \%)$.

\section{Targeted Specimen Analysis}

Specimens were obtained from all the visible lesions detected during the surveillance colonoscopies, either as resected lesions or in the form of targeted biopsies. There was a total of 62 neoplastic lesions. These neoplastic visible lesions were found in 34 patients (21.0\%).

The histopathological evaluation of the neoplastic lesions revealed the presence of LGD in 59, HGD in 2, and $\mathrm{ADC}$ in 1 . Of the 59 lesions with LGD, 4 were patients referred for surgery ( 3 underwent proctocolectomy and 1 underwent transanal resection after refusing a proctocolectomy) after being deemed unfit for endoscopic resection due to the nodular/irregular appearance/undefined limits of the lesions ( 3 cases) or multifocal lesions ( 1 case). The analysis of the surgical specimens confirmed the presence of LGD in 3 patients (including the transanal resection) and multifocality in 1 . In the remaining patient, pathology revealed the presence of an adenocarcinoma.

The 55 remaining lesions with LGD were considered endoscopically resectable and were further classified as either polypoid or nonpolypoid. Forty-one were polypoid (39 sessile and 2 pedunculated), 24 of which were removed with biopsy forceps (lesions $<5 \mathrm{~mm}$ ), 13 with coldsnare polypectomy, and 4 with hot-snare polypectomy. Fourteen lesions were classified as nonpolypoid (all superficially elevated) and were removed using an endoscopic mucosal resection technique. A biopsy was performed around the area of the polypectomy in $49.1 \%$ of the cases and these were all negative for dysplasia.

The HGD and ADC lesions were treated surgically (by proctocolectomy). The 2 lesions with HGD were detected in the same patient and the surgical specimen revealed the presence of synchronous ADC of the colon. Postoperative histopathology of the lesion with ADC in the targeted biopsy confirmed the presence of ADC.

\section{Random Biopsy Analysis}

For the random biopsy analysis, only the 4 random biopsies performed at every $10 \mathrm{~cm}$ were considered. Biopsies performed in the areas surrounding resected lesions were excluded.

Dysplasia in random biopsies was present in 3 patients (LGD in all). The dysplasia yield of random biopsies was $1.85 \% /$ patient (3/162), $8.8 \%$ /patient with dysplasia $(3 / 34)$, $1.75 \% /$ colonoscopy $(6 / 342)$, and $0.25 \% /$ biopsy $(9 / 3,637)$.

The colonoscopies in which dysplasia was found on random biopsy for the first time during the study period were performed using standard-definition scopes and WLE without CE. Regarding the endoscopic findings in the 3 patients: 1 presented simultaneously with a dysplas- 
Table 3. Univariate and multivariate logistic regression analyses evaluating associations between risk factors and dysplasia in at least one of the exams (either visible or from random biopsies)

\begin{tabular}{|c|c|c|c|c|}
\hline Clinical and endoscopic factors & $\begin{array}{l}\text { Univariate analysis } \\
\text { OR }(95 \% \mathrm{CI})\end{array}$ & $p$ value & $\begin{array}{l}\text { Multivariate logistic regression analysis } \\
\text { OR }(95 \% \mathrm{CI})\end{array}$ & $p$ value \\
\hline Gender & $2.0(0.9-4.4)$ & 0.077 & - & - \\
\hline Type of IBD (CD vs. UC) & $0.3(0.1-0.8)$ & 0.016 & $0.5(0.2-1.6)$ & 0.266 \\
\hline UC disease extent (left vs. pancolitis) & $0.5(0.2-1.4)$ & 0.186 & - & - \\
\hline PSC & $1.9(0.3-11.0)$ & 0.607 & - & - \\
\hline A family history of CRC & $0.4(0.1-2.0)$ & 0.368 & - & - \\
\hline A personal history of dysplasia & $1.9(0.3-11.0)$ & 0.607 & - & - \\
\hline Random biopsies performed & $9.7(1.3-73.8)$ & 0.009 & $7.7(0.9-64.6)$ & 0.061 \\
\hline CE performed & $2.1(1.0-4.6)$ & 0.05 & $1.1(0.5-2.7)$ & 0.780 \\
\hline 5-Aminosalicylic acid & $1.6(0.2-14.0)$ & 1.00 & - & - \\
\hline Thiopurines & $0.6(0.2-1.2)$ & 0.131 & - & - \\
\hline Anti-TNF therapy & $0.6(0.2-1.6)$ & 0.324 & - & - \\
\hline Colonic strictures & $0.9(0.9-1.0)$ & 0.347 & - & - \\
\hline Postinflammatory polyps & $0.9(0.4-2.0)$ & 0.837 & - & - \\
\hline Disease duration in years & - & - & $1.0(1.0-1.1)$ & 0.030 \\
\hline
\end{tabular}

$\mathrm{CD}$, Crohn's disease; CE, chromoendoscopy; CRC, colorectal cancer; PSC, primary sclerosing cholangitis; UC, ulcerative colitis.

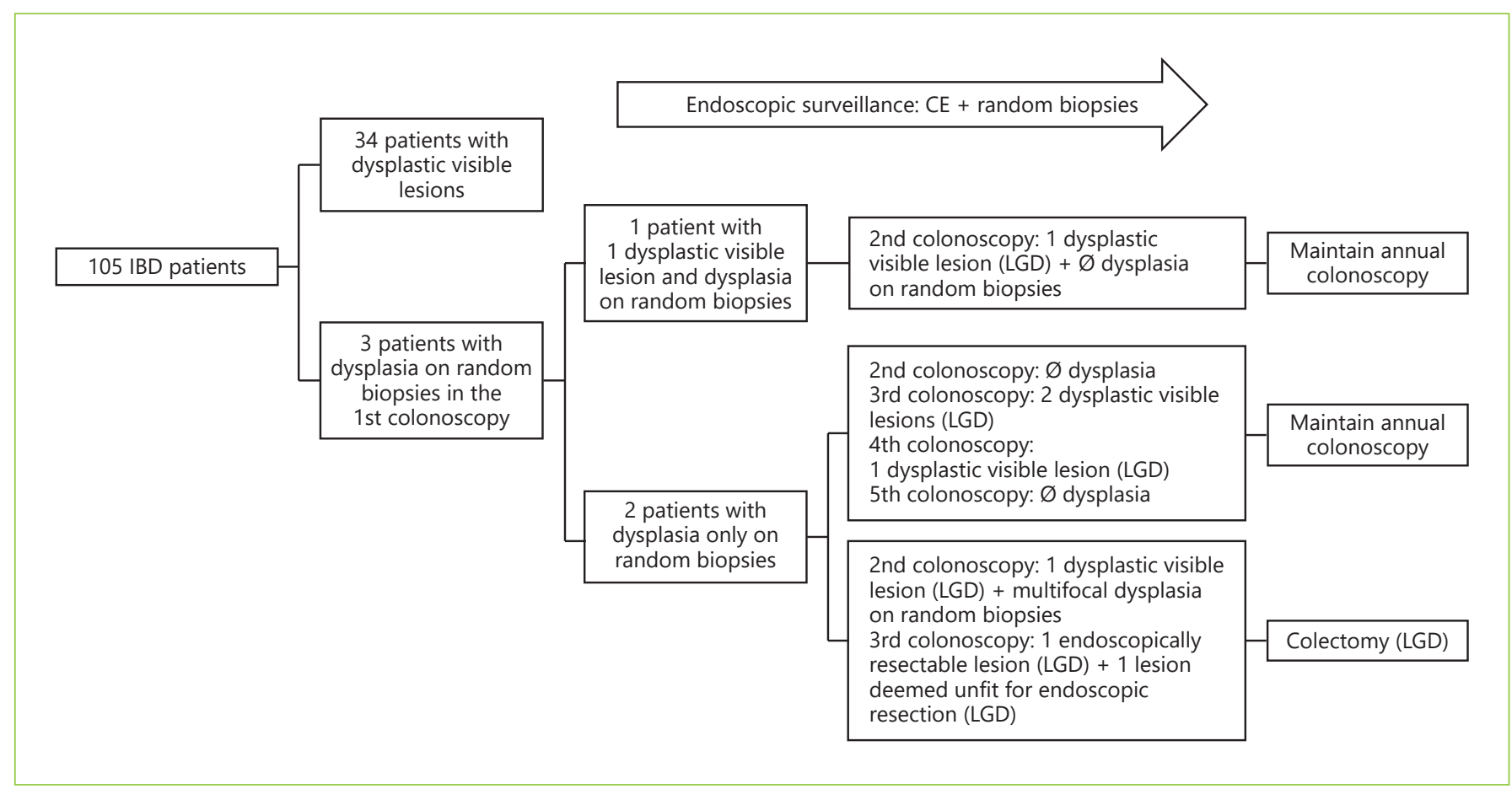

Fig. 1. Outcome of patients with dysplasia in random biopsies. CE, chromoendoscopy; IBD, inflammatory bowel disease; LGD, low-grade dysplasia.

tic visible lesion and dysplasia on random biopsy in the first surveillance colonoscopy, but dysplasia was found only on the random biopsy in the other 2 (multifocal in 1) during the first colonoscopy of the surveillance pro- gram. Therefore, the per-patient dysplasia yield of random biopsies alone was 5.9\% (2/34). Regarding disease activity, in 2 of the patients, there was no active disease; in 1 patient, moderate IBD activity was present. 


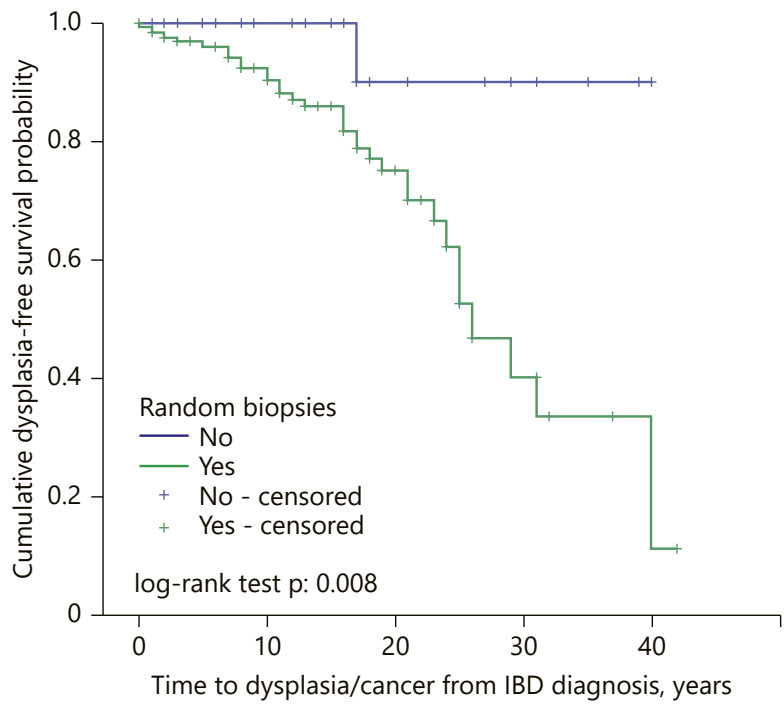

Fig. 2. Kaplan-Meier curve of time to dysplasia/cancer in patients in whom random biopsies were performed versus patients who did not undergo random biopsies.

All patients chose to be kept under surveillance and underwent annual CE plus random biopsies. The outcomes for the patients with dysplasia in random biopsies appear in Figure 1.

\section{Results of Univariate Analysis and Multivariate}

Logistic Regression

Upon univariate analysis, the finding of dysplasia (either visible or in random biopsies) at least once during the study period was significantly associated with the type of IBD (26.7\% in UC vs. $10.5 \%$ in CD) ( $p=0.016)$, performing random biopsies $(p=0.009)$, and performing $\mathrm{CE}$ $(p=0.05)$ (Table 3).

Detection of dysplasia on random biopsies was significantly associated with a personal history of visible dysplasia $(p=0.006)$.

Median disease duration was significantly longer in patients with at least 1 finding of dysplasia than in those without dysplasia (14.0 [IQR 5.75-21.0] vs. 9.0 [IQR $3.25-15.0]$ years; $p=0.03)$. No significant association was found between a finding of dysplasia and the extent of UC disease, a family history of CCR or a personal history of PSC, the presence of colonic strictures or postinflammatory polyps, or the use of medication (Table 3 ).

On multivariate analysis, considering the type of IBD, disease duration, and the performance of $\mathrm{CE}$ or random biopsy at least once, only duration of disease was found
Table 4. Cox regression analysis: factors associated with dysplasia detection

\begin{tabular}{llll}
\hline Endoscopic factors & Hazard ratio & $95 \%$ CI & $p$ value \\
\hline Random biopsies & 8.81 & $1.2-66.5$ & 0.035 \\
Chromoendoscopy & 1.08 & $0.5-2.2$ & 0.835 \\
\hline
\end{tabular}

to be independently associated with dysplasia $(p=0.03)$ (Table 3). The performance of random biopsies tended to an association with the finding of dysplasia at least once $(p=0.06)$.

\section{Results of the Survival Analysis}

The survival analysis showed there was no statistically significant difference in the time to dysplasia/cancer distribution for patients who underwent CE or not $\left(\chi^{2}{ }_{1}=\right.$ $1.429, p=0.23$ ) but the survival distributions were significantly different between patients who underwent random biopsies at least once and those who did not $\left(\chi^{2}{ }_{1}=\right.$ $7,115, p=0.008$ ) (Fig. 2). The mean time to dysplasia/ cancer in the random biopsies group was 27.4 (95\% CI 23.9-30.8) years, shorter than in the group without random biopsies at 37.7 (95\% CI 33.4-42.0).

There were $70.4 \%$ of censored cases in the CE group and $83.3 \%$ in the group without CE, 5.0\% in the random biopsies group, and $96.7 \%$ in the group without biopsies.

When a multivariate Cox regression analysis was done, including both the performance of $\mathrm{CE}$ and of random biopsies, the latter was still significantly associated with time to dysplasia/cancer (hazard ratio [HR] 8.81; 95\% CI $1.2-66.5 ; p=0.035)($ Table 4$)$.

\section{Discussion}

This study analyzed the clinical and endoscopic variables associated with dysplasia in IBD patients who were part of a colonoscopy surveillance program. A total of 362 colonoscopies were performed on 162 IBD patients.

Dysplasia was found at least once in 34 patients. The overall yield of dysplasia was $21.0 \%$, which is in the upper limit of what has been published in the literature (i.e., between 6 and 21\%) $[10,11,17]$. This outcome may be due to various factors. First, one should consider that our hospital is a tertiary referral center that specializes in oncology and may therefore deal with a higher percentage of high-risk patients. Besides the high risk in the cohort, the high rate of detection of neoplasia in our work may have 
been related to the fact that expert endoscopists, with extensive experience in the $\mathrm{CE}$ technique, performed the endoscopic procedures. Moreover, targeted and random biopsies were performed, not only when using standarddefinition colonoscopy, but also in $95 \%$ of the cases where CE was applied.

Regarding the risk factors for dysplasia, we found that patients with UC had a higher risk than CD patients, but only in the univariate analysis. Although the risk of CRC is traditionally associated with long-standing UC, growing evidence suggests a similar risk in patients with $\mathrm{CD}$ and extensive colonic involvement [10]. The fact that more than two-thirds of our cohort had UC may have influenced our results. Therefore, we still support that the same surveillance strategy should be applied to both UC and CD patients with colonic involvement of long duration.

As expected from previous studies, disease duration was significantly associated with the probability of finding dysplasia or cancer, in both the univariate and multivariate analyses, and this is the main factor to take into account when assigning patients to surveillance programs.

Multiple studies have demonstrated a markedly greater yield of dysplasia detection with CE than with standard techniques $[4,10,11,18]$.

We also showed that detecting dysplasia was independently associated with CE (OR 2.1; 95\% CI 1.0-4.6; $p=$ $0.05)$ in the univariate analysis. This association did not remain significant in the multivariate analysis, but this could possibly be due to the low percentage of patients that underwent CE.

Performing random biopsies was also associated with the detection of dysplasia in the univariate analysis, and it almost reached significance in the multivariate analysis. In the survival analysis, this factor influenced the time to detection of dysplasia/cancer, but this must be treated with caution, because the difference in the percentage of censored cases between groups was $>10 \%$.

Invisible dysplasia was found in 3 patients on the first surveillance colonoscopy. Dysplasia was classified as LG in all cases. In two of these patients, there was no active disease; in the other, moderate IBD activity was present. In this last case, inflammation may have confounded both the endoscopic and the histological diagnosis. However, in the subsequent surveillance examinations, dysplastic visible lesions were found. The fact that in all 3 cases, the exams were performed using WLE and standard-definition colonoscopes without $\mathrm{CE}$ should make us question if this dysplasia was indeed truly "invisible". Still, as shown in Figure 1, in 1 of these patients, multifocal invisible dysplasia was again found when CE was used.

In all 3 patients, the finding of dysplasia on random biopsy led to the shortening of the surveillance interval to 1 year. In the subsequent endoscopic evaluations, dysplastic visible lesions were detected in all patients. We may speculate that if the random biopsies hadn't been performed, at least 2 of these patients (i.e., with no synchronous visible lesions) would have been kept under larger surveillance intervals ( 5 years in the case of extensive colitis with no other risk features, and 2-3 years in the case of extensive colitis with moderate active inflammation), and more advanced lesions might have developed.

The need for random biopsies during the surveillance of IBD patient has been intensively debated among experts. In the SCENIC study, panelists did not reach consensus on this subject when using high-definition WLE or chromoendoscopy [8].

Arguments against the performance of random biopsy include its low yield, particularly when using CE or other advanced endoscopic techniques which improve neoplasia detection. Moreover, the increased duration of the endoscopic procedure and the costs associated with the pathology analysis of the specimens are considered excessive $[8,10,11,18]$. The clinical impact of performing random biopsies was evaluated in a retrospective study conducted by van den Broek et al. [11]. They concluded that neoplasia detected by random biopsy only rarely led to an altered clinical management. Although they showed a lack of clinical impact of this procedure, they nevertheless recommend performing random biopsies in the presence of specific risk factors, namely, in cases of PSC, tubular colon, and visible neoplastic lesions.

In the same way, Moussata et al. [10] reiterate in their study the importance of performing random biopsies in association with CE in patients with a personal history of neoplasia, PSC, or a tubular-appearing colon. They base their results on the fact that dysplasia in patients with IBD may begin in the crypt bases, and then progress with time to involve the full length of the crypt and the surface epithelium (the bottom-up growth pattern), explaining why CE may fail in detecting early dysplasia.

One of the major strengths of this study is that it reinforces the impact of random biopsies during surveillance colonoscopy in specific groups of patients, particularly when standard-definition colonoscopes are used. Similar to in the studies cited above $[10,11]$, a personal history of visible dysplasia $(p=0.006)$ was independently associated with dysplasia detected on random biopsies. Therefore, 
we underline that, although the clinical impact of random biopsies is generally low, these should not be totally omitted, but rather used selectively.

Based on our findings, we agree with the guidelines stating that $\mathrm{CE}$ and high-definition colonoscopy should be used for surveillance in all patients with long-standing IBD. The option to perform only targeted biopsies may then be enough for most patients. However, our study relaunches the importance of random biopsies under certain circumstances. Based on our results, we consider that random biopsies should always be done when $\mathrm{CE}$ is not possible, when standard definition colonoscopes are used or in the presence of inflammation or high-risk features. Namely, when there is a personal history of dysplasia, random biopsies should be added to CE to increase the dysplasia detection rate. Additionally, based on published data, patients with concurrent PSC or tubular-appearing colon should also be considered as being at high risk and random biopsies should be performed in these patients even when CE is used [10].

Our study has some limitations, namely the small number of patients included, with a low number of patients with PSC or a family history of CRC. This may explain the lack of a significant association between these established risk factors and dysplasia in our study. Although our surveillance protocol is well established, the planned CE was only achieved in one-third of the patients, mainly due to inadequate bowel cleanliness. In the future, measures should be implemented to optimize bowel preparation, allowing for $\mathrm{CE}$ in all patients included in the surveillance program. In a few cases, colonoscopy was performed by different experienced IBD endoscopists, especially at the beginning of the study. This may also have contributed to a lack of uniformity of the methods used. Currently, this limitation has been overcome because one experienced endoscopist performs all the exams.

The quality of the bowel preparation could not be included in our analysis because, in the earlier years of the study, no standard validated bowel preparation quality scale was used. Suboptimal preparations may affect the dysplasia detection rate and we could not account for this factor. Likewise, disease activity was not always described using standard classifications early in the study, so we could not account for it in our analysis.

As already stated, another major limitation of this study was the use of standard-definition scopes. In fact, according to the SCENIC study, high definition is recommended, since it improves visualization and enhances dysplasia detection $[8,18]$. Our conclusions may not apply to high-definition colonoscopy.
The duration of the procedure and the presence of a tubular-appearing colon, factors that may also affect the detection/presence of dysplasia, were not consistently registered and could therefore not be studied. A prospective registration of all these data is currently being done and may clarify results in the future.

Finally, we did not perform a cost-effectiveness analysis; costs should also be taken into account when planning surveillance programs.

\section{Conclusion}

This study evaluated the clinical and endoscopic variables associated with dysplasia in IBD patients over a 6 -year surveillance period. Our data confirm that patients with long-standing IBD, particularly UC, should be enrolled in dysplasia surveillance programs. Moreover, although our data were limited to standard definition colonoscopies, we have, again, shown that performing CE in dysplasia surveillance programs in IBD patients helps in the detection of colonic neoplastic lesions. Our results are in agreement with authors defending the notion that random biopsies should not be abandoned, but rather used selectively in certain groups of patients.

\section{Statement of Ethics}

The authors have no ethical conflicts to disclose. All patients gave their written consent.

\section{Conflict of Interest Statement}

I.R. reports grants, personal fees, or nonfinancial support from ABBVIE, FERRING, MSD, TAKEDA, PHARMAKERN, JANSSEN, and DR FALK PHARMA, outside the submitted work. The remaining authors have nothing to disclose.

\section{Funding Sources}

There was no funding.

\section{Author Contributions}

S.S.: study design, data collection, and drafting of the manuscript; I.R.: study design, performance of endoscopic examinations, and revision of the manuscript; J.M.: revision of the manuscript; J.P.d.S.: revision of the manuscript; R.F.: performance of histopathological analysis and revision of the manuscript; A.D.P.: critical revision and final approval of the manuscript. 


\section{References}

1 Eaden JA, Abrams KR, Mayberry JF. The risk of colorectal cancer in ulcerative colitis: a meta-analysis. Gut. 2001 Apr;48(4):526-35.

2 Andersen NN, Jess T. Has the risk of colorectal cancer in inflammatory bowel disease decreased? World J Gastroenterol. 2013 Nov; 19(43):7561-8.

3 Beaugerie L, Svrcek M, Seksik P, et al. Risk of colorectal high-grade dysplasia and cancer in a prospective observational cohort of patients with inflammatory bowel disease. Gastroenterology 2013; 145:166-75.

4 Magro F, Gionchetti P, Eliakim R, Ardizzone S, Armuzzi A, Barreiro-de Acosta M, et al.; European Crohn's and Colitis Organisation [ECCO]. Third European Evidence-based Consensus on Diagnosis and Management of Ulcerative Colitis. Part 1: Definitions, Diagnosis, Extra-intestinal Manifestations, Pregnancy, Cancer Surveillance, Surgery, and Ileo-anal Pouch Disorders. J Crohns Colitis. 2017 Jun; 11(6):649-70.

5 Jess T, Simonsen J, Jørgensen KT, Pedersen BV, Nielsen NM, Frisch M. Decreasing risk of colorectal cancer in patients with inflammatory bowel disease over 30 years. Gastroenterology. 2012 Aug;143(2):375-81.e1.

6 Ten Hove JR, Bernstein CN, Oldenburg B. Putting Evidence into Practice: IBD Surveillance, Chromoendoscopy and Future Directions. Am J Gastroenterol. 2018 Mar;113(3): 313-6.

7 Iacucci M, Kaplan GG, Panaccione R, Akinola O, Lethebe BC, Lowerison M, et al. A Randomized Trial Comparing High Definition
Colonoscopy Alone with High-Definition Dye Spraying and Electronic Virtual Chromoendoscopy for Detection of Colonic Neoplastic Lesions during IBD Surveillance Colonoscopy. Am J Gastroenterol. 2018 Feb; 113(2):225-34.

8 Laine L, Kaltenbach T, Barkun A, McQuaid KR, Subramanian V, Soetikno R; SCENIC Guideline Development Panel. SCENIC international consensus statement on surveillance and management of dysplasia in inflammatory bowel disease. Gastroenterology. 2015 Mar;148(3):639-651.e28.

9 Watanabe T, Ajioka Y, Mitsuyama K, Watanabe K, Hanai H, Nakase H, et al. Comparison of targeted vs random biopsies for surveillance of ulcerative colitis-associated colorectal cancer. Gastroenterology. 2016 Dec; 151(6):1122-30.

10 Moussata D, Allez M, Cazals-Hatem D, Treton X, Laharie D, Reimund JM, et al.; the GETAID. Are random biopsies still useful for the detection of neoplasia in patients with IBD undergoing surveillance colonoscopy with chromoendoscopy? Gut. 2018 Apr; 67(4):616-24.

11 van den Broek FJ, Stokkers PC, Reitsma JB, Boltjes RP, Ponsioen CY, Fockens P, et al. Random biopsies taken during colonoscopic surveillance of patients with longstanding ulcerative colitis: low yield and absence of clinical consequences. Am J Gastroenterol. 2014 May; 109(5):715-22.

12 Navaneethan U, Kochhar G, Venkatesh PG Bennett AE, Rizk M, Shen B, et al. Random biopsies during surveillance colonoscopy increase dysplasia detection in patients with primary sclerosing cholangitis and ulcerative colitis. J Crohn's Colitis. 2013 Dec;7(12):97481.

13 Lewis JD, Chuai S, Nessel L, et al. Use of the noninvasive components of the Mayo score to assess clinical response in ulcerative colitis. Inflamm Bowel Dis 2008; 14(12):1660-6.

14 Daperno M, D’Haens G, Van Assche G, Baert F, Bulois P, Maunoury V, et al. Development and validation of a new, simplified endoscopic activity score for Crohn's disease: the SESCD. Gastrointest Endosc. 2004 Oct;60(4): 505-12.

15 Rutgeerts P, Geboes K, Vantrappen G, Beyls J, Kerremans R, Hiele M. Predictability of the postoperative course of Crohn's disease. Gastroenterology. 1990 Oct;99(4):956-63.

16 Dixon MF. Gastrointestinal epithelial neoplasia: vienna revisited. Gut. 2002 Jul;51(1):130 1 .

17 Marion JF, Waye JD, Present DH, Israel Y, Bodian C, Harpaz N, et al.; Chromoendoscopy Study Group at Mount Sinai School of Medicine. Chromoendoscopy-targeted biopsies are superior to standard colonoscopic surveillance for detecting dysplasia in inflammatory bowel disease patients: a prospective endoscopic trial. Am J Gastroenterol. 2008 Sep;103(9):2342-9.

18 Verdon C, Aruljothy A, Lakatos PL, Bessissow T. Endoscopic surveillance strategies for dysplasia in ulcerative colitis. Frontline Gastroenterol. 2019 Apr;11(2):124-32. 